Binghamton University

The Open Repository @ Binghamton (The ORB)

Mechanical Engineering Faculty Scholarship

Mechanical Engineering

2011

\title{
A Large-Stroke Electrostatic Micro-Actuator
}

Shahrzad Towfighian

Binghamton University--SUNY, stowfigh@binghamton.edu

Abdulrahman Seleim

University of Windsor, seleim@uwindsor.ca

Eihab Abdel-Rahman

University of Waterloo, eihab@uwaterloo.ca

Glenn Heppler

University of Waterloo, heppler@uwaterloo.ca

Follow this and additional works at: https://orb.binghamton.edu/mechanical_fac

Part of the Mechanical Engineering Commons

\section{Recommended Citation}

Towfighian, Shahrzad; Seleim, Abdulrahman; Abdel-Rahman, Eihab; and Heppler, Glenn, "A Large-Stroke Electrostatic Micro-Actuator" (2011). Mechanical Engineering Faculty Scholarship. 11.

https://orb.binghamton.edu/mechanical_fac/11

This Article is brought to you for free and open access by the Mechanical Engineering at The Open Repository @ Binghamton (The ORB). It has been accepted for inclusion in Mechanical Engineering Faculty Scholarship by an authorized administrator of The Open Repository @ Binghamton (The ORB). For more information, please contact ORB@binghamton.edu. 


\title{
A large-stroke electrostatic micro-actuator
}

\author{
S. Towfighian ${ }^{1 *}$, A. Seleim ${ }^{2 \times}$, E. M. Abdel-Rahman ${ }^{3+}$, and G. \\ R. Heppler ${ }^{4+}$ \\ * Mechanical and Industrial Engineering, University of Toronto, Toronto, Ontario, \\ Canada. $\times$ Industrial and Manufacturing Systems Engineering, University of \\ Windsor, Ontario, Canada. + System Design Engineering, University of Waterloo, \\ Waterloo, Ontario, Canada. \\ E-mail: ${ }^{1}$ sherryt@mie.utoronto.ca, ${ }^{2}$ seleim@uwindsor.ca, ${ }^{3}$ corresponding \\ author: eihab@uwaterloo.ca, and ${ }^{4}$ heppler@uwaterloo.ca
}

\begin{abstract}
Voltage driven parallel-plate electrostatic actuators suffer from an operation range limit of $30 \%$ of the electrostatic gap; this has restrained their application in microelectromechanical systems (MEMS). In this paper, the travel range of an electrostatic actuator made of a micro-cantilever beam above a fixed electrode is extended quasi-statically to $90 \%$ of the capacitor gap by introducing a voltage regulator (controller) circuit designed for low frequency actuation. The voltage regulator reduces the actuator input voltage, and therefore the electrostatic force, as the beam approaches the fixed electrode so that balance is maintained between the mechanical restoring force and the electrostatic force. The low-frequency actuator also shows evidence of high order superharmonic resonances that are observed here for the first time in electrostatic actuators.
\end{abstract}




\section{Introduction}

Extending the range of electrostatic actuation is desirable in many applications including MEMS optical switches [1], tunable laser diodes [2], polychromator gratings [3], optical modulators [4], and millipede data storage systems [5]. To extend the travel range of electrostatic actuators in attracting mode beyond the conventional one-third of the capacitor gap, researchers have used various methods including: charge and current control [6-9], and leveraged bending [10]. Other approaches that seek to extend the travel range of electrostatic actuators include voltage control [11-13], sliding mode control [14], and other nonlinear feedback controllers [15-17].

Charge and current control are very difficult to perform in MEMS because of the very small capacitance. Using a capacitor in series with the actuator to control the charge, Chan et al. [9] experimentally increased travel up to $60 \%$ of the gap at the cost of high voltage requirements. Adding a capacitor in series, they kept the electrostatic force constant in the actuator by controlling the voltage difference between the actuator plates. As the gap decreases in the actuator beyond $30 \%$ of the gap, its capacitance increases rapidly, while the voltage across the actuator plates decreases as the square of the gap helping to keep the electrostatic force constant. Theoretically, it should have been possible to operate over the full gap of the actuator, but parasitic capacitance limited the travel range to $60 \%$ of the gap. Leveraged bending [10] is another method to increase the actuation range by applying the electrostatic force only to a portion of the actuator. The disadvantage of this method is high input voltage requirements.

Current control was examined by Guardia et al. [8]. They used open loop and closed loop configurations for driving the actuator with a current source. They showed full gap actuation is achievable with a voltage five times the open-loop pull-in voltage.

Experimentally they obtained actuation up to $48 \%$ of the gap. The drawback of this method was that the actuator was stable only for a short period of time.

There are a number of studies using charge control for extending electrostatic actuation [6,7]. Using a switched-capacitor circuit Seeger et al. [6] controlled the charge of an electrostatic actuator. They showed analytically that the pull-in instability can be eliminated by taking advantage of parasitic capacitance present in the actuator and employing a voltage 5.2 times smaller than that of voltage controlled systems. They experimentally achieved $83 \%$ of the gap. However, tip-in instability, which arises from a small rotation of the parallel plate actuator, and snapping could not be eliminated using this technique.

Using voltage control Chen et al. [12] stabilized the tilt angle of an electrostatic micro-mirror beyond its snap-down angle. They control the actuation voltage, keeping the slope of the mechanical torque larger than the electrostatic torque to stabilize the angle of the mirror. They were able to extend the stable operation range to $10^{\circ}$ from an open-loop pull-in angle of $6.1^{\circ}$. Simulation studies correlated well with experiments at low actuation angles; at large actuation angles the linear mechanical and damping force models could not predict the overshoot accurately. 
Employing a nonlinear voltage controller that uses two control methods, feedback linearization and trajectory planning, Agudelo et al. [13] experimentally achieved actuation up to $60 \%$ of the gap, well beyond pull-in, for an electrostatic micro-mirror. They used feedback of the mirror tilt angle and the actuation voltage to follow the desired angle trajectory beyond pull-in and obtained good agreement with simulation. Simulation showed actuation over the whole gap, this was limited in practice by the sampling time which restricted the actuation to $60 \%$ of the gap.

A nonlinear output tracking controller was proposed by Owusu et al. [16] to extend the electrostatic actuation range using displacement, velocity and charge feedback. Simulations showed robust response in the presence of noise and an increased actuation range to $90 \%$ of the gap. Further studies on this controller were conducted by Nikpanah et al. [17] who improved the performance by eliminating the displacement fluctuations present in the previous study when using a switching control technique that made the controller more feasible to implement.

A closed loop controller that used a capacitive sensor for position feedback was designed by Lu and Fedder [11] for position tracking of probe-based magnetic disk drives. They added a constant controller gain that ensured a minimum phase margin of $60^{\circ}$ to a linearized model of the actuator and experimentally stabilized the actuator up to $60 \%$ of the gap. Liu et al. [18] used a lumped mass model for the cantilever beam to study the probe response and reported chaotic oscillation in the presence of forced excitations. The cantilever beam mode shapes were then used and a comprehensive study of the nonlinear system dynamics was carried out by Towfighian et al. [19] to investigate the controller parameters for stable and bi-stable behaviors, and chaotic oscillations.

The largest quasi-static micro-actuation range reported so far is limited to 60 $\%$ of the capacitor gap for closed loop actuators and $30 \%$ of the gap for open loop actuators. Parallel-plate electrostatic actuators able to traverse the entire capacitor gap with reasonable actuation voltage are an important addition to the state-of-the-art since they will open the doors to wide spread use of these high-energy density actuators. For this purpose, we develop a large-stroke actuator by applying a simplified control law to a micro-cantilever beam. We study the quasi-static and dynamic response of the largestroke actuator experimentally and numerically to characterize its performance. In the following sections, we describe the closed-loop actuator model and report the numerical and experimental performance of the actuator in quasi-static and dynamic actuation modes, respectively.

\section{Actuator Model}

The actuator consists of a polysilicon cantilever beam as a moving electrode above a fixed electrode (Figure 1). A picture of the fabricated PolyMUMPS actuator is shown in Figure 2. A feedback control system is added to the open loop electrostatic actuator to extend the stable electrostatic actuation range. 


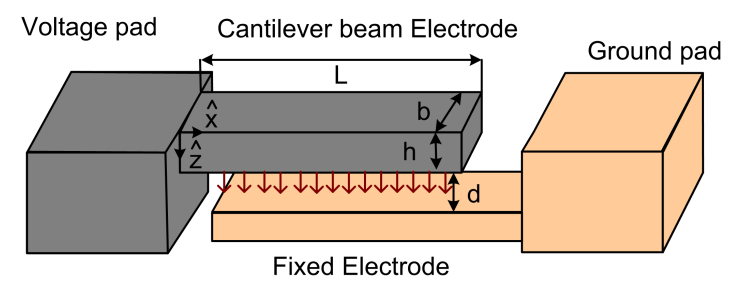

Figure 1. Schematic of micro-beam oscillator.

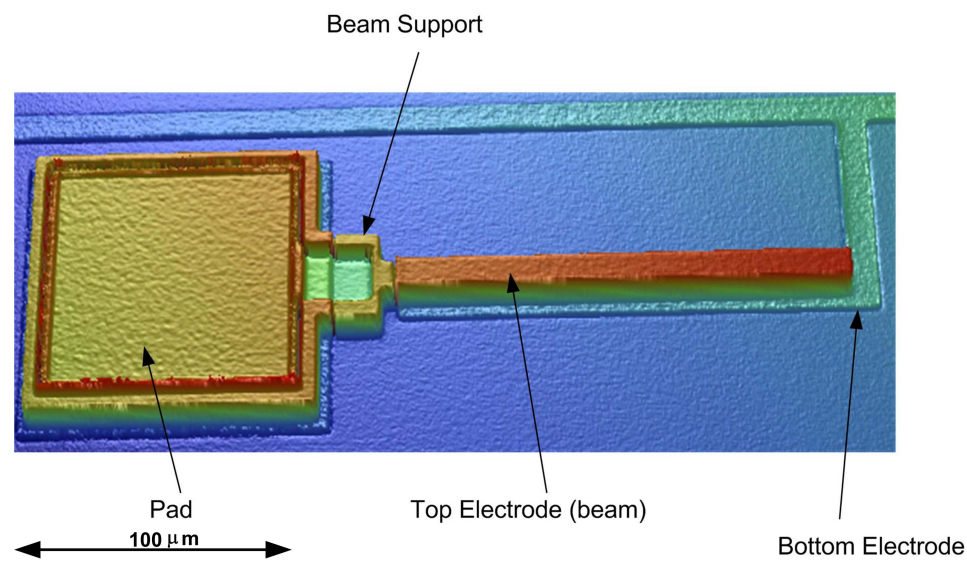

Figure 2. A white-light image of the electrostatic actuator obtained with a WYKO NT1100 Optical Profiler.

The controller regulates the actuator voltage, and therefore the electrostatic force, to obtain a larger range of motion. A schematic of the closed-loop system is shown in Figure 3. In this figure, $V_{i n}$ is the input voltage, $V_{c}$ is the controller output voltage, $G$ is a voltage gain, and $\hat{w}$ is the deflection of the beam in the $\hat{z}$ direction (Figure 1 ). The voltage applied between the two electrodes (see Figure 3) creates the electrostatic forcing on the cantilever beam

$$
F_{e} \propto G^{2} \frac{\left(V_{i n}-V_{c}\right)^{2}}{(d-\hat{w})^{2}},
$$

where $d-\hat{w}$ is the gap.

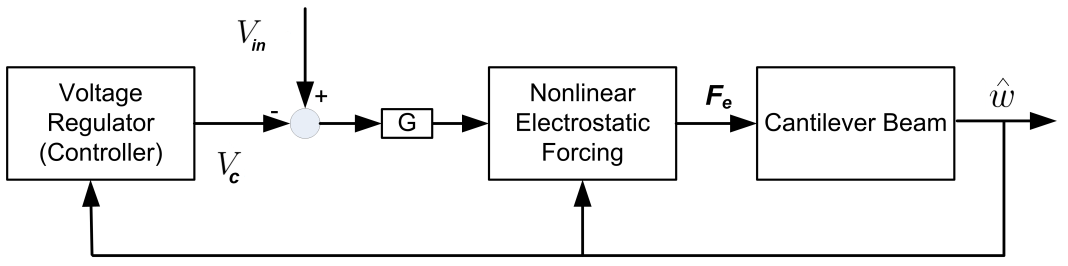

Figure 3. Closed loop system. 
The equation of motion of the closed loop is [19]

$$
\rho A \frac{\partial^{2} \hat{w}(\hat{x}, \hat{t})}{\partial \hat{t}^{2}}+E I \frac{\partial^{4} \hat{w}(\hat{x}, \hat{t})}{\partial \hat{x}^{4}}+c \frac{\partial \hat{w}(\hat{x}, \hat{t})}{\partial \hat{t}}=\frac{\varepsilon_{0} b G^{2}\left(V_{i n}-V_{c}\right)^{2}}{2(d-\hat{w}(\hat{x}, \hat{t}))^{2}}
$$

where $A$ is the cross sectional area of the beam, $E I$ is the flexural rigidity of the beam, the coefficient $c$ accounts for damping losses due to the beam motion through the air. Other system parameters are identified experimentally as described in Towfighian et al. [20] and are given in Table 1 . To generalize the equation of motion, we use the following non-dimensional variables to re-write Eq. (2):

$$
x=\frac{\hat{x}}{L}, w=\frac{\hat{w}}{d}, t=\frac{\hat{t}}{T}
$$

where the time constant $T$ is

$$
T=\sqrt{\frac{\rho A L^{4}}{E I}} .
$$

We multiply the result by the denominator of the electrostatic force term to obtain

$$
\ddot{w}(1-w)^{2}+w^{(4)}(1-w)^{2}+\mu \dot{w}(1-w)^{2}=G^{2} \alpha\left(V_{i n}-V_{c}\right)^{2} .
$$

where

$$
\mu=\frac{c L^{4}}{E I T}, \alpha=\frac{\varepsilon_{0} b L^{4}}{2 E I d^{3}},
$$

and $w^{(4)}$ is the fourth derivative of deflection with respect to the axial coordinate $x$. To transform Eq. (5) to an ordinary differential equation, it is discretized using separation of variables and Galerkin's method with the trial function chosen as the first mode shape of the cantilever beam. The set of ordinary differential equations of the actuator including the controller equation is then

$$
\left\{\begin{array}{l}
\left(\ddot{q}+\mu \dot{q}+\omega_{1}^{2} q\right)\left(1+c_{1} q+c_{2} q^{2}\right)=c_{3} G^{2} \alpha\left(V_{i n}-V_{c}\right)^{2} \\
\dot{V}_{c}=-r\left(V_{c}-\frac{q}{1-q} \Psi\right)
\end{array}\right.
$$

where $q$ is the beam tip deflection normalized with respect to the initial gap, over-dot means differentiation with respect to time, $\omega_{1}$ is the first natural frequency of the beam, and $c_{1}, c_{2}$, and $c_{3}$ are found by applying Galerkin's method. The parameters appearing in the differential equation for the controller voltage $\left(V_{c}\right)$ [18] are the voltage gain $G$, displacement gain $\Psi$, and controller damping $r$.

Typically in applications such as AFM based memory devices or in programmable optical filters [3], actuators travel from an undeflected position to a static hold at a target location. In these applications resonant and high frequency oscillations are not advantageous. Henceforth, choosing an excitation frequency around one fourteenth of the resonant frequency, large stable actuations are investigated quasi-statically. Restricting the tests to low frequency allows simplification of the control circuit. The controller Equation (7) can be rewritten in the Laplace domain as

$$
s V_{c}=-r V_{c}+r \Psi \frac{q}{1-q}
$$


Table 1. Actuator parameters

\begin{tabular}{|l|c|c|}
\hline Parameter & Symbol & Value \\
\hline Displacement Gain & $\Psi$ & $0.5 ~ V$ \\
\hline Voltage Gain & $G$ & 4.8 \\
\hline Controller Damping & $\mathrm{r}$ & 100 \\
\hline Density of polysilicon & $\rho$ & $2331 \mathrm{~kg} / \mathrm{m}^{3}$ \\
\hline Beam Length & $L$ & $131 \mu \mathrm{m}$ \\
\hline Beam Width & $b$ & $20 \mu \mathrm{m}$ \\
\hline Beam Thickness & $h$ & $1.9 \mu \mathrm{m}$ \\
\hline Initial gap & $d$ & $1.9 \mu \mathrm{m}$ \\
\hline Nondimensional Damping coefficient & $\mu$ & 0.157 \\
\hline Permittivity of air & $\varepsilon$ & $8.85 \times 10^{-12} \mathrm{~F} / \mathrm{m}$ \\
\hline Modulus of Elasticity & $E$ & $150 \mathrm{GPa}$ \\
\hline
\end{tabular}

and after rearranging this becomes

$$
\frac{V_{c}}{\Psi \frac{q}{1-q}}=\frac{1}{1+\frac{s}{r}}
$$

The righthand side of Eq. (9) is a low pass filter with a nondimensional cut off frequency of $r$. When operating at nondimensional frequencies much lower than the cut off frequency $\hat{\Omega} \ll r$ (or dimensional frequencies $\Omega \ll r / T$ ), the ratio $\frac{s}{r}$ in Eq. (9) approaches zero and thus it can be simplified to

$$
V_{c} \approx \Psi \frac{q}{1-q}
$$

Using the previous equation to generate the control voltage simplifies the implementation of the controller and allows for a more compact device. The revised system equations are then obtained as

$$
\left\{\begin{array}{l}
\left(\ddot{q}+\mu \dot{q}+\omega_{1}^{2} q\right)\left(1+c_{1} q+c_{2} q^{2}\right)=c_{3} \alpha G^{2}\left(V_{i n}-V_{c}\right)^{2} \\
V_{c}=\frac{q}{1-q} \Psi
\end{array}\right.
$$

The stable actuator is obtained by setting the voltage regulator parameters to obtain a stable equilibrium point for most of the gap distance [19], Figure 4 (b). The results in part (b) of the figure were obtained by numerically solving the algebraic equations obtained by equating the time derivatives in Eq. (11) to zero. In this figure, the solid line shows the location of the stable equilibrium point and the dashed line shows the location of the unstable saddle point. While the stable equilibrium point defines the rest position of the beam, the stable manifold of the saddle delineates the basin of safe oscillations around that equilibrium position. The static profile reveals that the controller displaces the saddle away from the stable equilibrium point; this is revealed by comparing to the open loop static response (Figure 4 (a)). The increased distance of the saddle from the stable equilibrium point creates larger basin for safe motions to evolve around the equilibrium point. 

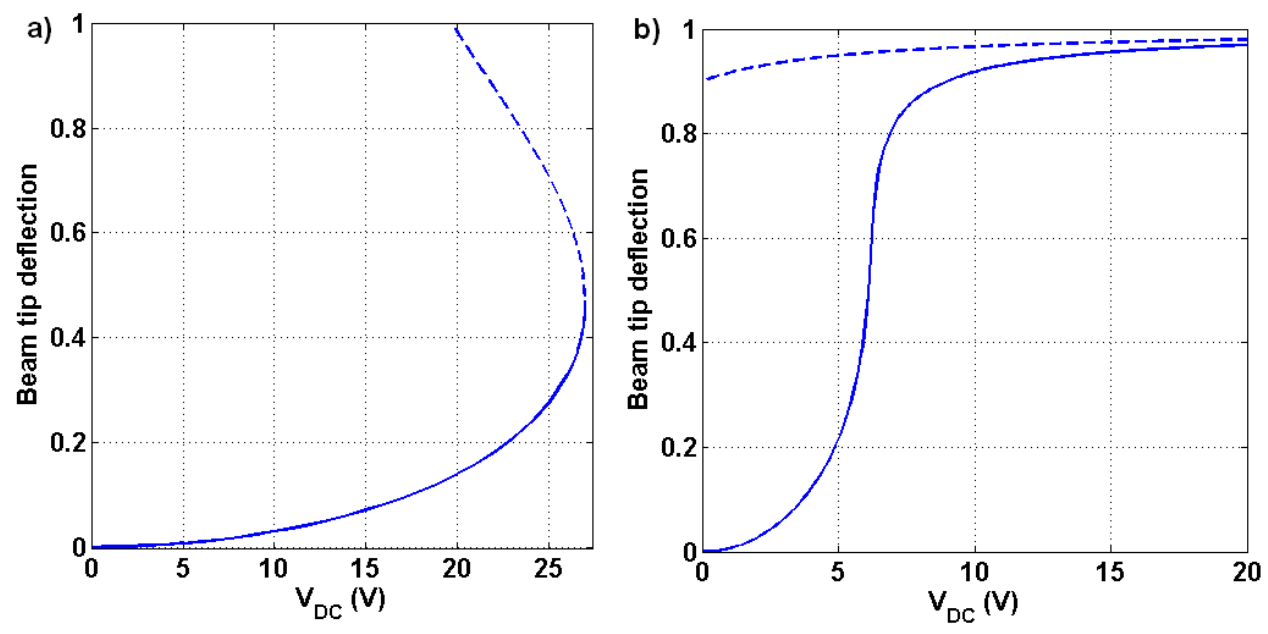

Figure 4. a) Open loop displacement b) Closed loop displacement. Dashed lines show the location of unstable equilibrium points (saddles) and solid lines show the stable equilibrium location.

\section{Quasi-static Actuation}

\subsection{Closed Loop System}

The closed loop system is implemented by introducing a circuit that acts as a voltage regulator. The controller function, the second of Eqs. (11), was implemented using analog electronics [21]. Despite the advantages of digital control such as easy code changes, an analog system is chosen because of its fast response. A challenge with analog systems though, is the difficulty in changing the controller function. The voltage regulator input was provided by a signal function generator, and the regulator op-amps were operated using two power supplies. The intermediate parameters were measured along the circuit and were displayed on the oscilloscope.

A schematic of the closed loop system is shown in Figure 5 and further details can be found in reference [22]. First, the beam tip velocity $\dot{q}$ is measured with the vibrometer, the corresponding signal is then passed through a high pass filter, cut off at $30 \mathrm{~Hz}$, to eliminate ground vibration generated by the vacuum pump used to hold the chip in place. Next, the filtered velocity signal is integrated in the circuit to find the beam tip displacement $q$. The displacement signal is scaled so that the entire electrostatic gap is equivalent to $1 \mathrm{~V}$. Next, the displacement signal is subtracted from a constant voltage of 1 to evaluate $(1-q)$, and division is performed to find $\frac{q}{1-q}$. The result is multiplied by the displacement gain $\Psi$ to find the control voltage $V_{c}$. This is subtracted from the input voltage $V_{i n}$, and the result is finally multiplied by the voltage gain $G$ to generate the regulated voltage $G\left(V_{i n}-V_{c}\right)$. The regulated voltage is applied to the actuator. This voltage can control the beam position in the stable regime to create a stable large-stroke actuator. The schematic of the control circuit is shown in Figure 6 , where subtraction and multiplication are implemented using op-amps and division is 
realized using diodes [21].

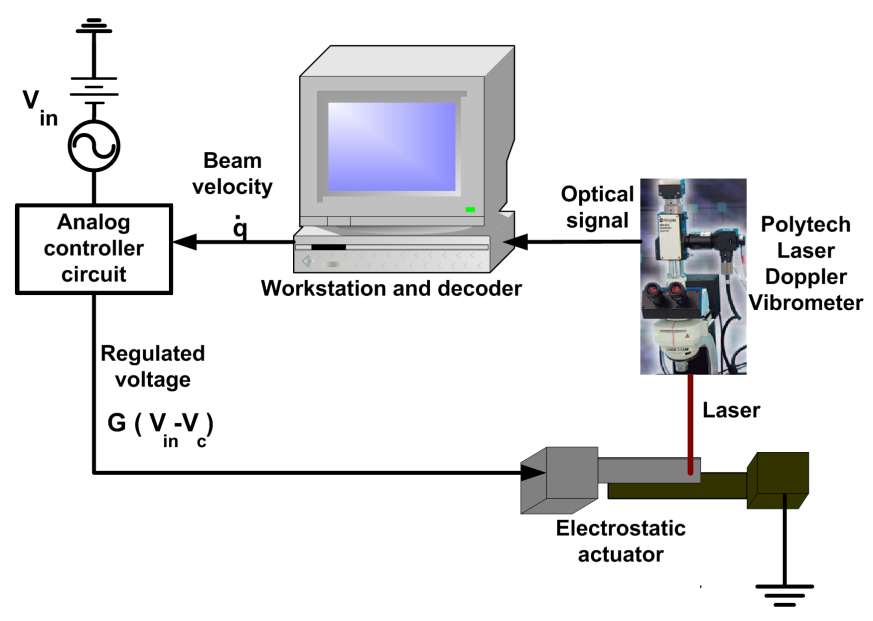

Figure 5. Schematic of the closed loop system.

The closed loop actuator was tested using a sinusoidal excitation voltage according to $V_{i n}=v(1+\cos (\Omega t))$ that varies between zero and a maximum voltage that corresponds to a desired static location in Figure 4 (b). The maximum excitation, $2 v$, corresponds to the horizontal axis of Figure 4, and the target location corresponds to the vertical axis. Figures 7 to 11 compare experimental and simulated dynamic responses revealing quasi-static actuation with target positions located at 37\%,52\%,67\%, $78 \%$ and $90 \%$ of the gap, respectively. Simulations were performed by numerically solving Eq. (11) using the beam parameters and the damping ratio in Table 1 identified in [20]. The frequency of excitation was set to $10 \mathrm{kHz}$. In comparison to the beam natural frequency of $140 \mathrm{kHz}$, the forced motion was quasi-static. The damping ratio was 0.157 , the pullin voltage was $25 \mathrm{~V}$, and the beam length, width, and thickness were $131 \mu \mathrm{m}, 20 \mu \mathrm{m}$, and $1.9 \mu \mathrm{m}$, respectively. The gap distance was perturbed around the nominal value so that the magnitude of the maximum velocity obtained in simulations was equal to that obtained the experimentally. Using this method, the capacitor gap was identified as $d=1.82 \mu \mathrm{m}$, and $1.9 \mu \mathrm{m}$ for the beams employed in the tests shown in Figures 7 , and $8-11$, respectively. 


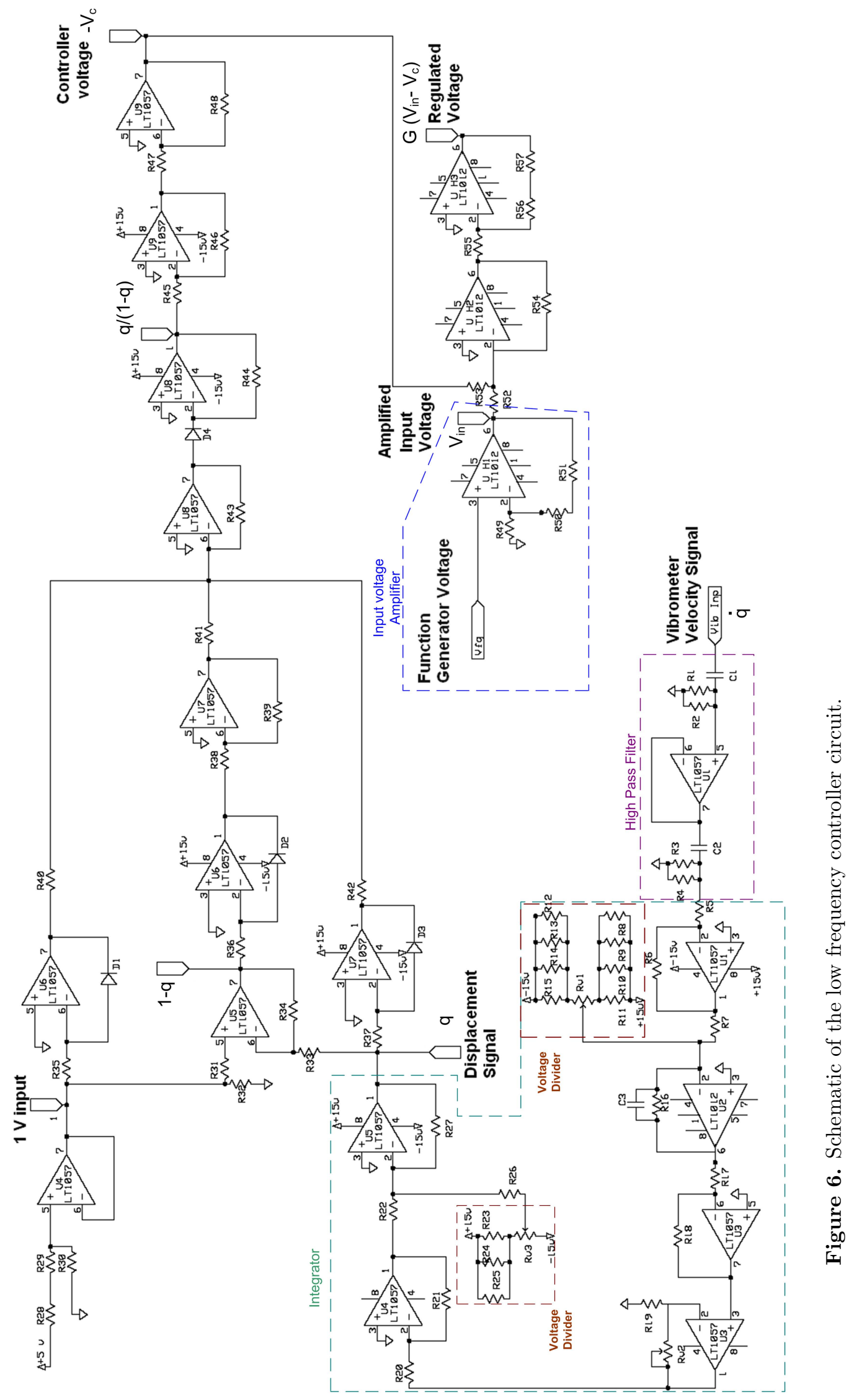



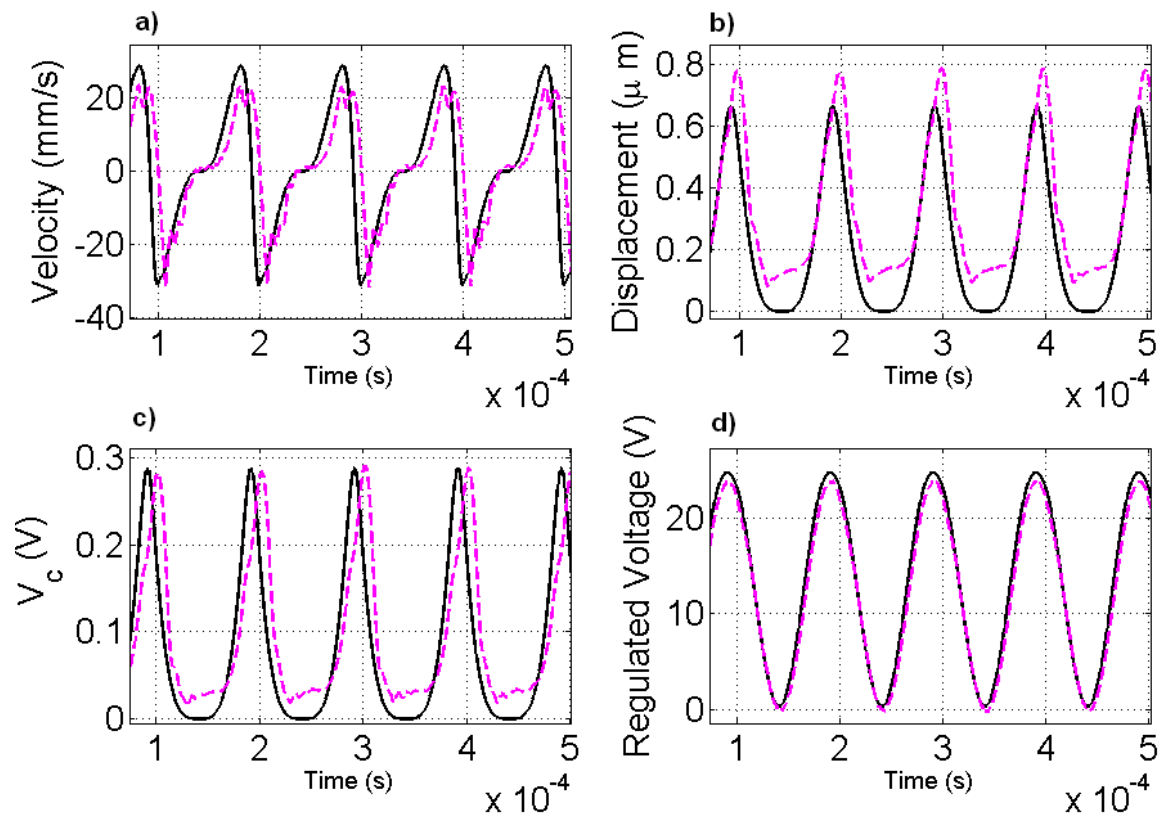

Figure 7. Closed loop response to an excitation of $v=2.7 \mathrm{~V}, \omega=10 \mathrm{kHz}$. - measured, - simulated. a) beam tip velocity, b) beam tip displacement, peak at $37 \%$, c) controller voltage $V_{c}$, d) Regulated voltage $G\left(V_{i n}-V_{c}\right)$. (Capacitor gap is $1.82 \mu \mathrm{m}$.)

The experimental results in Part (a) of the Figures present the velocity of the actuator tip measured by the vibrometer and Part (b) represents the displacement found from the integration of the velocity signal in the circuit. Part (c) and (d) show the measured controller voltage $V_{c}$, and the regulated voltage applied to the actuator $G\left(V_{i n}-V_{c}\right)$, respectively. The experimental results in parts (a) and part (b) of the figures are filtered in MATLAB by local regression using weighted linear least squares and a first degree polynomial model that assigns lower weight to outliers in the regression. The method assigns zero weight to data outside six mean absolute deviations.

Good agreement between simulation and measured responses is observed for a broad range of actuation voltage. The smallest range is presented in Figure 7, where though the controller voltage does not exceed $0.3 \mathrm{~V}$ and the regulated voltage does not go beyond the open loop pull-in voltage of $25 \mathrm{~V}$, the actuator traverses a trajectory that spans to $0.67 \mu \mathrm{m}$ or $37 \%$ of the gap which is greater than the $33 \%$ stability limit for open loop systems. The low signal to noise ratio for small displacements prevents the controlled motions from tracking the trajectory in the troughs. However, as the displacement increases and the signal to noise ratio improves, the controller follows the desired trajectory well. 

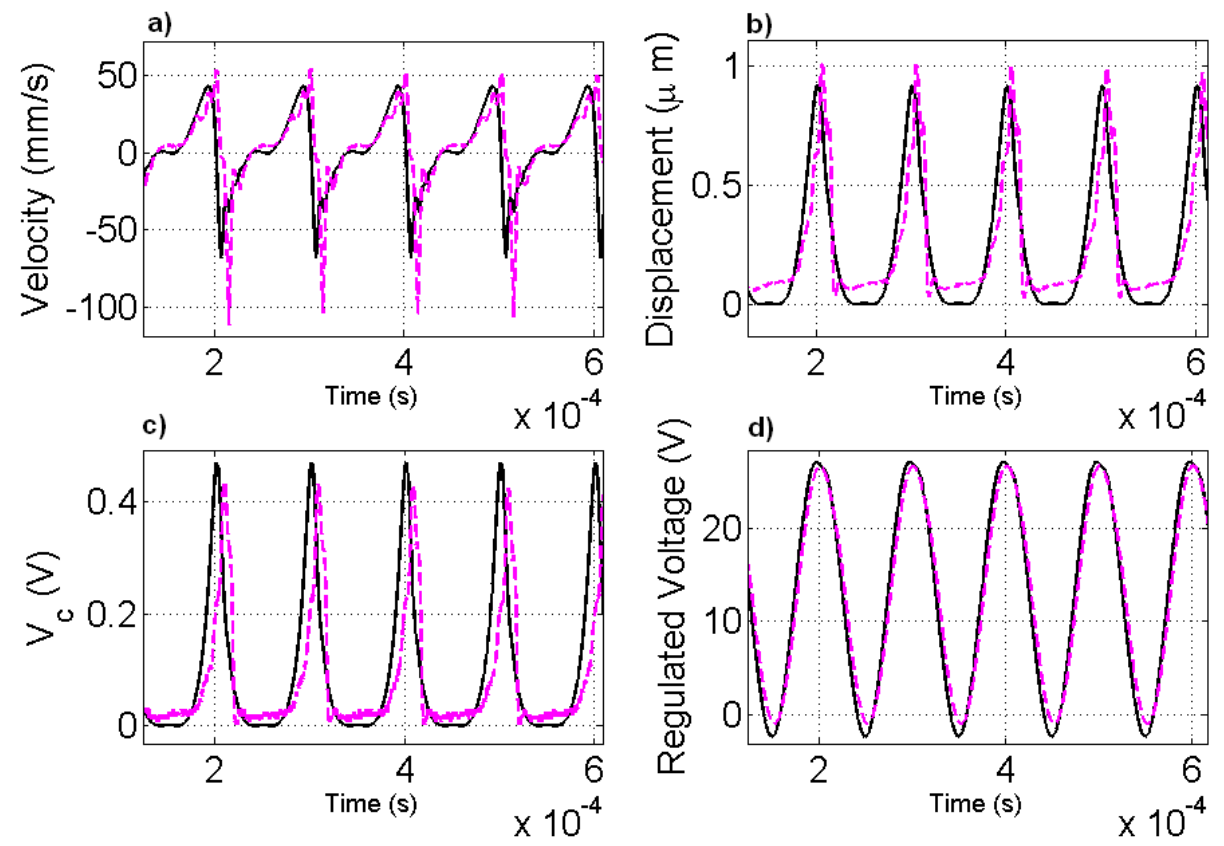

Figure 8. Closed loop response to excitation at $v=2.88 \mathrm{~V}, \omega=10 \mathrm{kHz}$. - measured, - simulated: a) beam tip velocity, b) beam tip displacement, peak at $52 \%$, c) controller voltage $V_{c}$, d) regulated voltage $G\left(V_{i n}-V_{c}\right)$. (Capacitor gap is $1.9 \mu \mathrm{m}$.)

Keeping the excitation frequency constant at $10 \mathrm{kHz}$ and increasing the peak voltage from $2.88 \mathrm{~V}$ to $3.2 \mathrm{~V}$ for a beam with a larger gap of $1.9 \mu \mathrm{m}$, the travel ranges increase from $52 \%(0.94 \mu \mathrm{m})$ to $90 \%(1.73 \mu \mathrm{m})$ in Figures 8 to 11 . This increase corresponds to a controller voltage rise from $0.45 \mathrm{~V}$ to $2.2 \mathrm{~V}$ as shown in part (c) of these figures and to the changes in the regulated voltage shape in part (d). The qualitative change in the regulated voltage originates from the increase of controller voltage $V_{c}$ in sub-figure (c) as the beam displacement reaches its maximum in sub-figure (b). Because the controller voltage peak and the input voltage peak have the same phase, there is a voltage drop at the peak of the regulated voltage across the capacitor $G\left(V_{i n}-V_{c}\right)$ shown in Figure $11(\mathrm{~d})$. The voltage drop at the peak of the regulated voltage plays an important role in stabilizing the actuator. As the actuation voltage increases in the open loop actuator, the electrostatic force increases causing more deflection in the beam which rapidly increases the electrostatic force even more. This positive feedback loop drives open-loop actuators to pull-in. The voltage regulator, on the other hand, interrupts this process by dropping the voltage and the electrostatic force as the displacement increases helping to balance it with the mechanical spring force and making large displacements feasible. At the maximum displacement, the actuator reverses direction in sub-figure (a) as the mechanical force dominates the electrostatic force and the beam returns to the undeflected position. It is noted that the signal to noise ratio in sub-figures (a) and (b) deteriorates as the input voltage increases, though that affects the oscillation that takes place around the undeflected position and not around the target large displacement 
points.
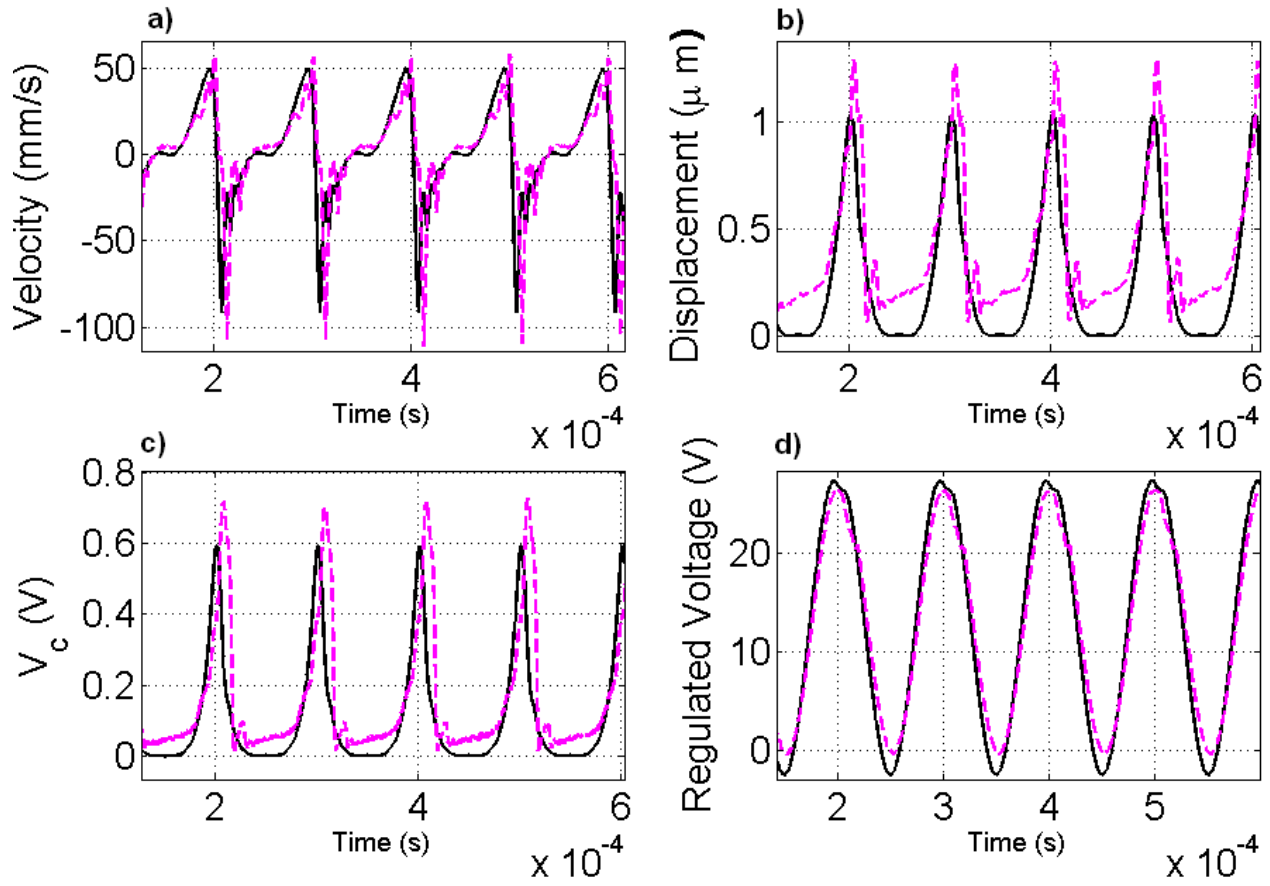

Figure 9. Closed loop response to excitation at $v=2.92 \mathrm{~V}, \omega=10 \mathrm{kHz}$. measured, - simulated. a) beam tip velocity, b) beam tip displacement, peak at $67 \%$, c) controller voltage $V_{c}$, d) Regulated voltage $G\left(V_{i n}-V_{c}\right)$. (Capacitor gap is 1.9 $\mu \mathrm{m}$.

The beam tip comes to within $400 \mathrm{~nm}$ and $100 \mathrm{~nm}$ of the substrate surface for the trajectories shown in Figures 10 and 11, respectively. This proximity is desirable for reading and writing in probe based high capacity data storage devices [11] using miniaturized AFM arrays.

Observing the displacement profiles, it is apparent that more oscillations occur as the actuator reverses its direction of motion away from the higher peaks and into the trough. These oscillations modulate the displacement and velocity profiles with oscillations at the natural frequency of the beam $\omega_{1}$. They occur because of the sudden motion reversal at the peak. On the other hand, the oscillations are not present at the counter motion reversal as the actuator climbs out of the trough because of our choice of input signal which guarantees a trough wide enough for the initial oscillations to die out and a gradual motion reversal. 

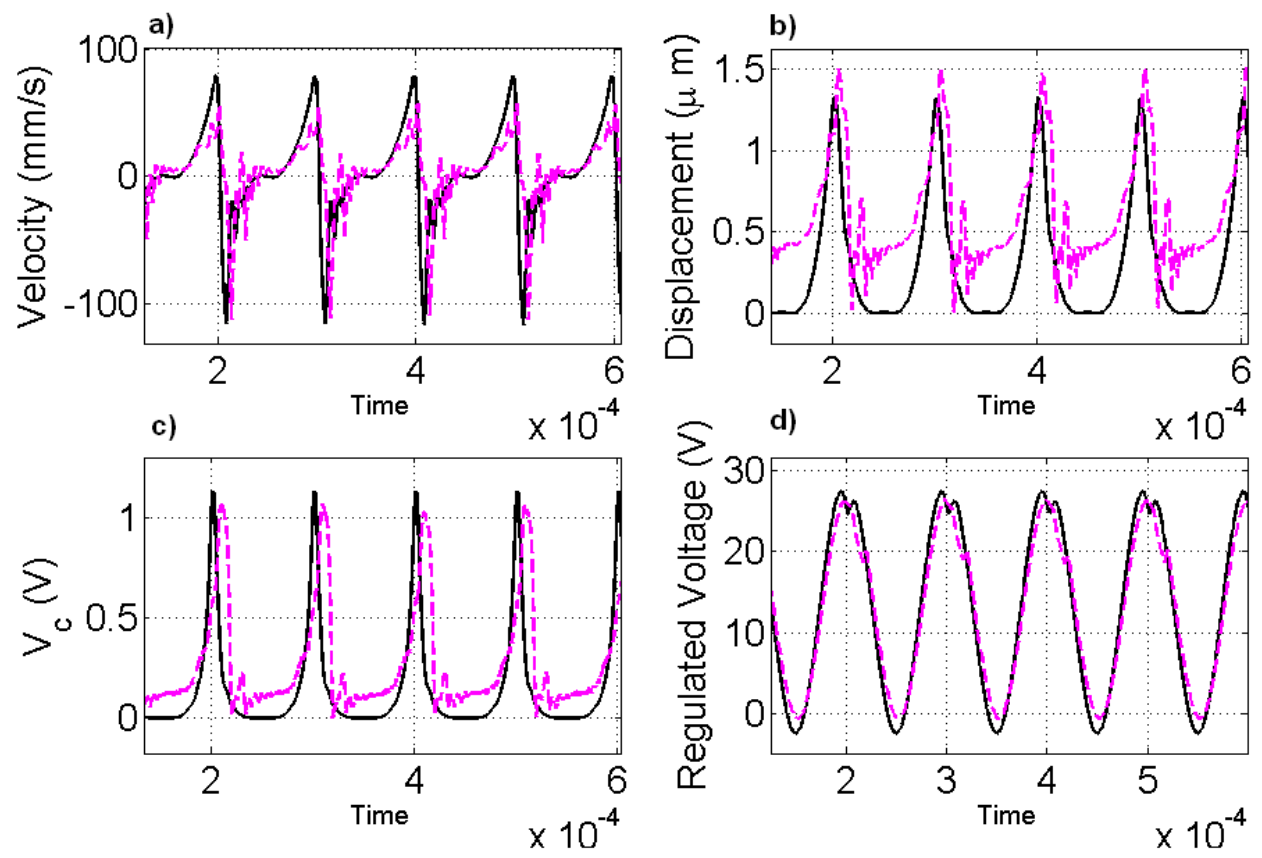

Figure 10. Closed loop response to excitation at $v=3 \mathrm{~V}, \omega=10 \mathrm{kHz}$. - - measured, - simulated. a) beam tip velocity, b) beam tip displacement, peak at $78 \%$, c) controller voltage $V_{c}$, d) regulated voltage $G\left(V_{i n}-V_{c}\right)$. (Capacitor gap is $1.9 \mu \mathrm{m}$.)

The modulated oscillations are not seen in the predicted path as the model uses a linear viscous damping term to represent the dissipation mechanisms in the actuator. In averaging out the highly nonlinear effects of squeeze-film damping, it overestimates the amount of damping present for the small oscillations in the trough around the undeflected position. The effects of the discrepancy between the linear damping model and the actual nonlinear damping effect are also clear in the simulated velocity matching the minimum measured negative velocity but not the maximum positive velocity in Figures 10 and 11. This difference between the simulated and measured velocities grows as the actuation range increases. These limitations not withstanding, the actuator reaches its target at the peak with minimal delay. Further, unlike the leveraged bending method, the regulated voltages does not exceed $27 \mathrm{~V}$ slightly larger than the pull-in voltage of $25 \mathrm{~V}$. 

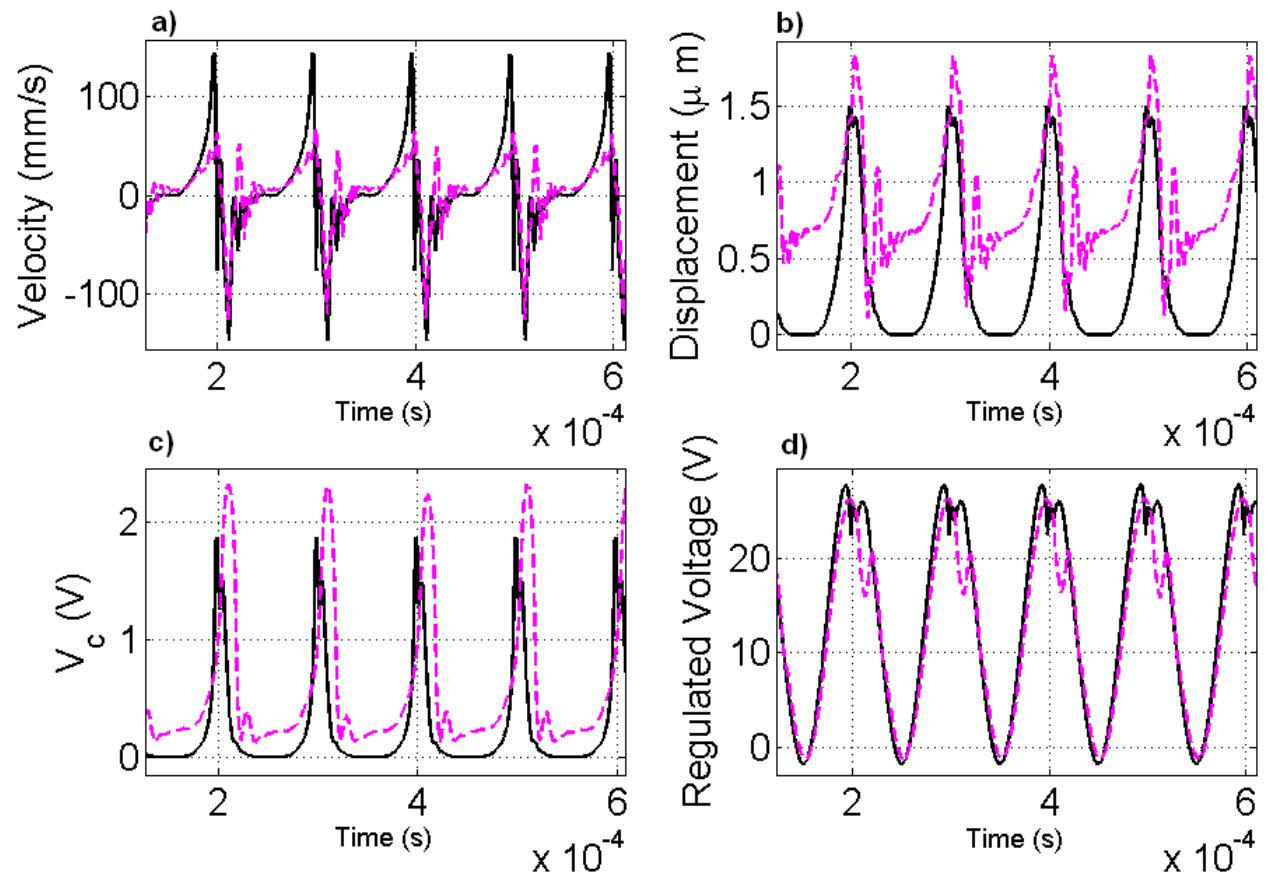

Figure 11. Closed loop response to excitation at $v=3.2 \mathrm{~V}, \omega=10 \mathrm{kHz}$. measured, - simulated: a) beam tip velocity, b) beam tip displacement, peak at $90 \%$, c) controller voltage $V_{c}$, d) regulated voltage $G\left(V_{i n}-V_{c}\right)$. (Capacitor gap is $1.9 \mu \mathrm{m}$.)

At the peak voltage drop, a small phase difference can be seen between the simulations and experiments in Figures 10(d) and 11(d) that is caused by the delays imposed by the op-amps in the controller circuit. The phase difference magnitudes are $8.6^{\circ}$ and $7.5^{\circ}$ respectively in those figures. This phase shift can be minimized using more precise non-inverting op-amps. However, actuation ranges are not affected by the controller phase delay.

The ultimate positions reached at different peak voltages are summarized in Figure 12 as well as the static deflection versus voltage curves shown earlier in Figure 4 (b). Figure 12 reveals a good agreement between the static simulation results and the quasistatic experimental measurements, thereby justifying the basic assumption underlying the simplified controller development. 


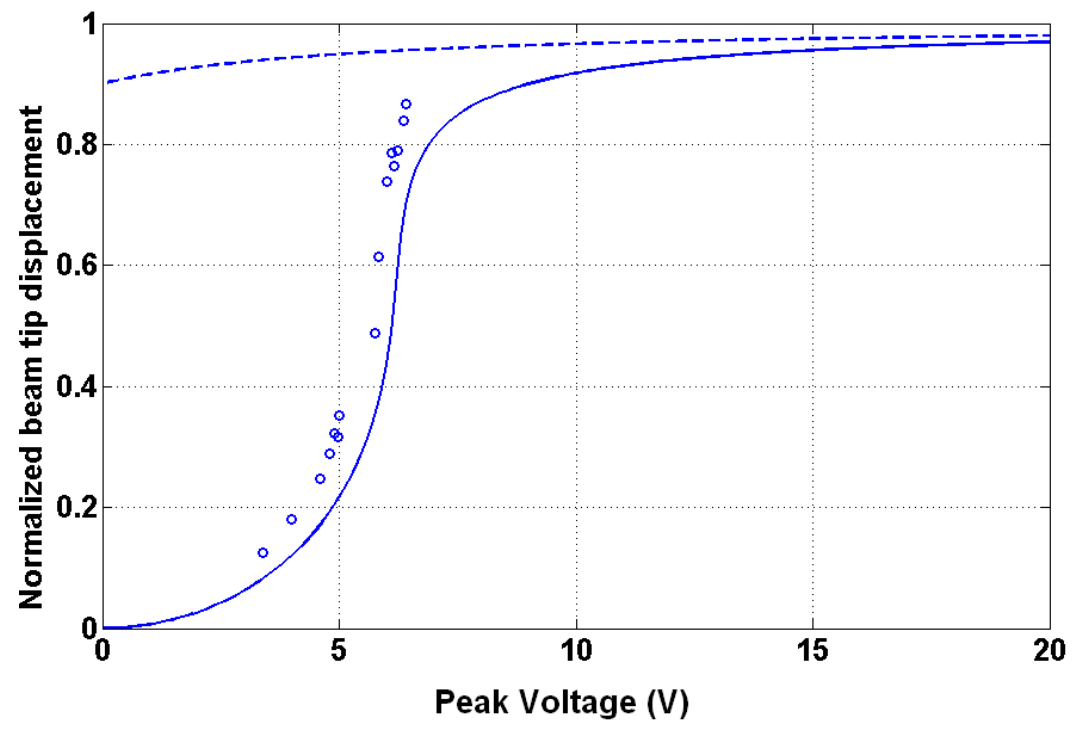

Figure 12. Peak actuator displacement versus peak voltage $(2 v)$. -- simulated unstable equilibrium points, - simulated stable equilibrium points, o experimental results. (Capacitor gap is $1.9 \mu \mathrm{m}$.)

\subsection{Virtual regulator}

Examining the response of the actuator to the controlled voltage, we postulated that applying the regulated voltage waveform obtained from the simulation directly to the beam without using the analog regulator could lead to similar results. The experimental setup consisted exclusively of the micro-beam, a signal generator, and a vibrometer to measure the beam tip velocity and an analog integrator. Simulations were performed using the closed loop control model to generate the regulated voltage signal. The signal generator was then used to produce the customized waveform, the beam response was measured using the vibrometer and the velocity was integrated using the analog integrator to find the beam displacement. The results are depicted in Figure 13, where the customized voltage signal is shown in part (c). Exciting the beam with the proposed waveform eliminates the need for the external feedback circuit while allowing for an actuation range as large as $75 \%$ of the gap which is significantly larger than the traditional open loop limit of $33 \%$ of the gap. Under this paradigm, the controller can be constructed as an off-line filter that can be used to filter out the components of the command voltage that drive the pull-in instability. 


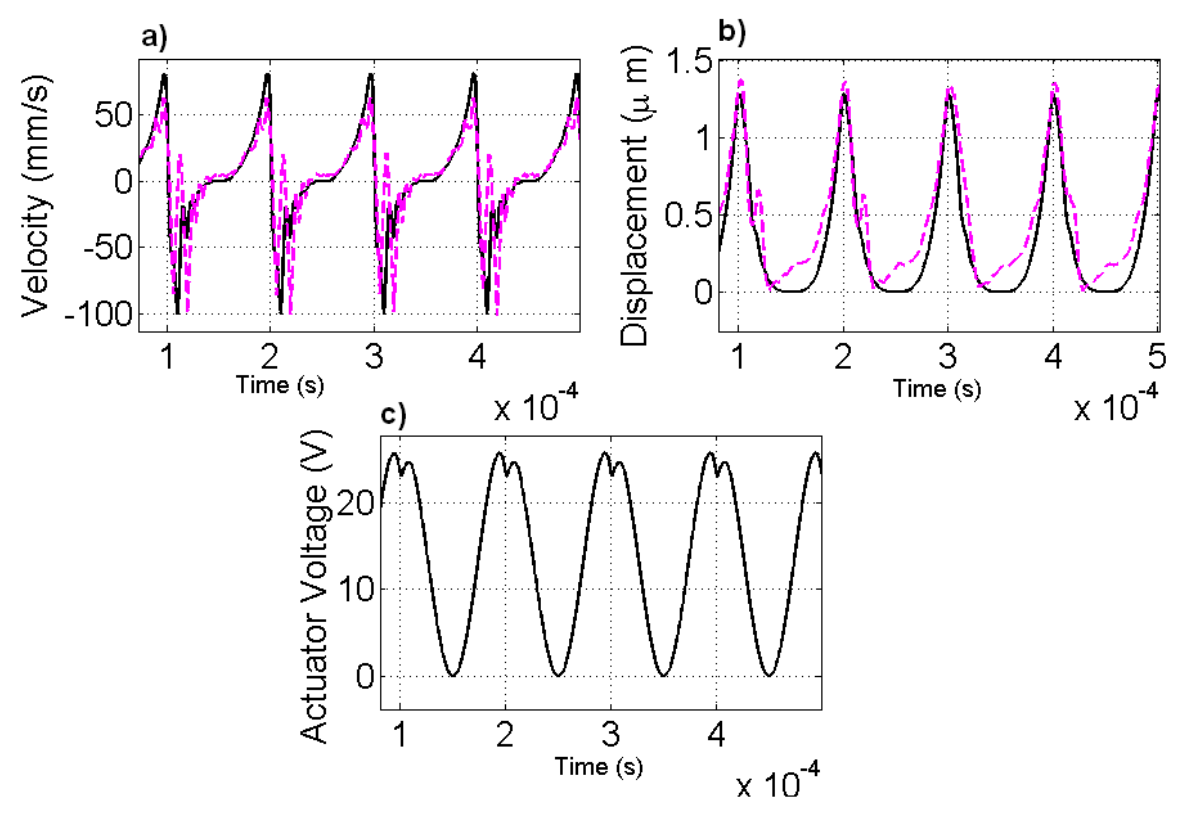

Figure 13. Actuator open loop response to a customized input waveform. -measured, - simulated response to excitation at $v=3 \mathrm{~V}, \omega=10 \mathrm{kHz}$ for a beam with dimensions of $131 \times 20 \times 1.9 \mu \mathrm{m}$ with the gap of $1.82 \mu \mathrm{m}$, and controller parameters of $\Psi=0.5 V, G=4.8$.

\section{Dynamic Actuation}

\subsection{Closed loop Superharmonic Resonances}

The closed loop actuator can also act as a resonator in regions of the frequency spectrum where superharmonic resonances appear. Exciting the system at one frequency, the system is responding at multiple frequencies. We studied the superharmonic resonances available at low frequency excitations $(<22 \mathrm{kHz})$. The results are presented in Figure 15 showing, on the left, the FFT of $2^{12}$ velocity data points and, on the right, the phase portrait obtained from plotting the measured velocity versus the integrated displacement. The beam under test has nominal dimensions of $175 \times 10 \times 2$ with a gap of 2 $\mu \mathrm{m}$ and a natural frequency of $80 \mathrm{kHz}$. All experiments were conducted on the same day to eliminate variation in the plant parameters due to aging and changes in temperature and humidity. The superharmonic resonances are verified by the FFT, where a train of FFT peaks extending from the excitation frequency to the natural frequency exists and the number of peaks reveals the order of the superharmonic resonance. The superharmonic resonances of order 8,6 , and 5 are reported at excitation frequencies of 10,13 , and $15.7 \mathrm{kHz}$ respectively with the same voltage excitation magnitudes $v=3.56 \mathrm{~V}$. The superharmonic orbits shown expand along the displacement and velocity axes as the order of the superharmonic drops from 8 to 5 . The stretching of the orbit along the displacement axis corresponds to higher dynamic amplification of the input at lower orders of the superharmonic resonance that leads to a higher signal 
to noise ratio.
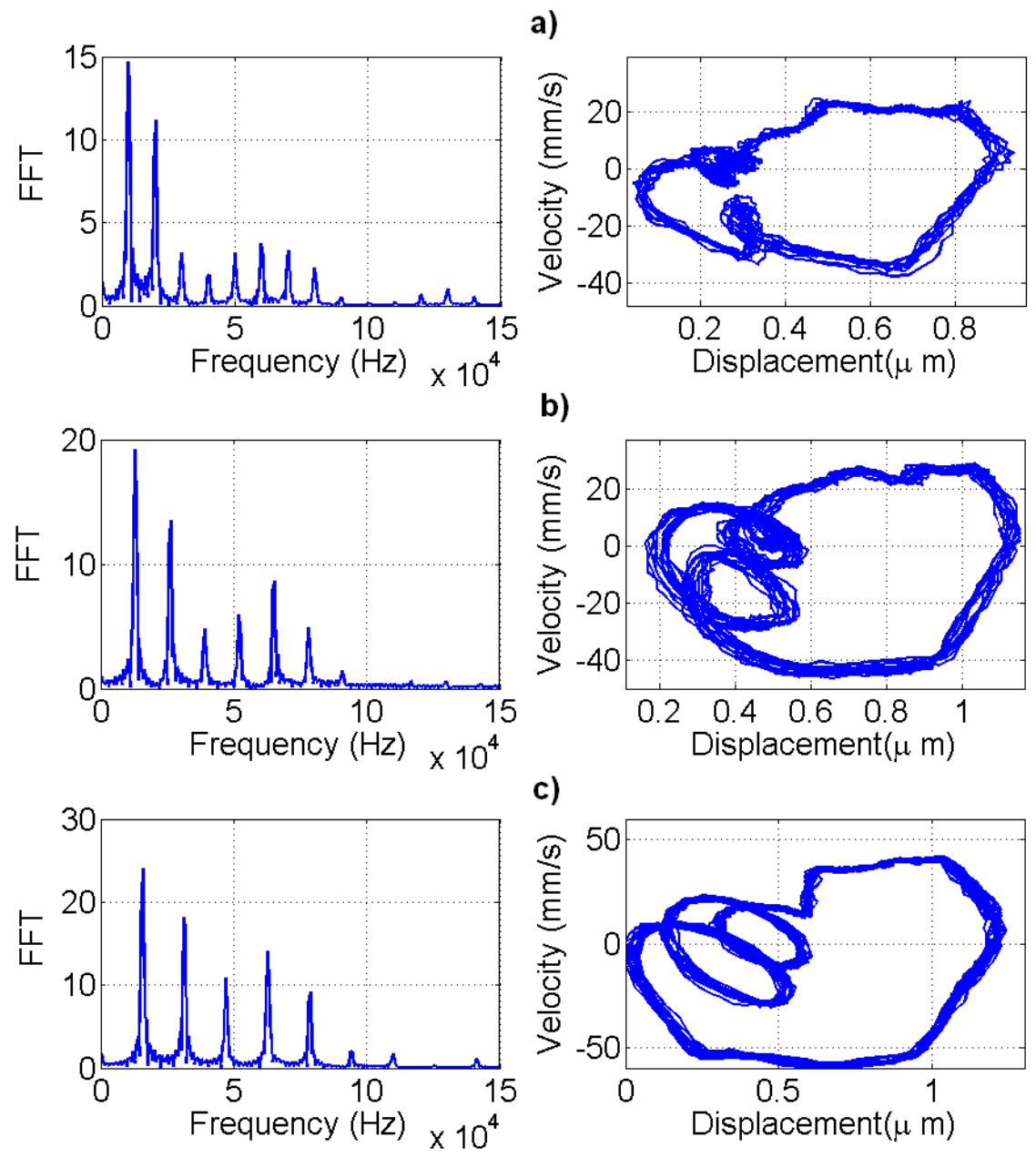

Figure 14. Fast Fourier Transform of the tip velocity and phase portrait for a beam with a natural frequency of $80 \mathrm{kHz}$ obtained when the controller parameters are $G=2, \Psi=0.5 \mathrm{~V}$, and the excitation amplitude is $v=3.56 \mathrm{~V}$ for the superharmonic resonances of a) order 8 at $\omega=10 \mathrm{kHz}, \mathrm{b}$ ) order 6 at $\omega=13 \mathrm{kHz}$, and c) order 5 at the excitation $\omega=15.7 \mathrm{kHz}$.

Testing a similar beam with a natural frequency of $85 \mathrm{kHz}$, the superharmonic resonance of order 4 was observed as shown in Figure 15 (d) at an excitation frequency of $21.36 \mathrm{kHz}$. Although the excitation voltage is lower than the previous set of tests, the phase portrait shows an orbit comparable with the previous three cases of superharmonic resonance. As the order of superharmonic resonances drops, the regularity increases and less harmonics appear in the FFT resulting in the reduction of the number of loops in the phase portrait. Although superharmonic resonances of order half, and two have been reported by Younis et al. [23], the first instance of higher order resonances in MEMS electrostatic actuators is observed here. 

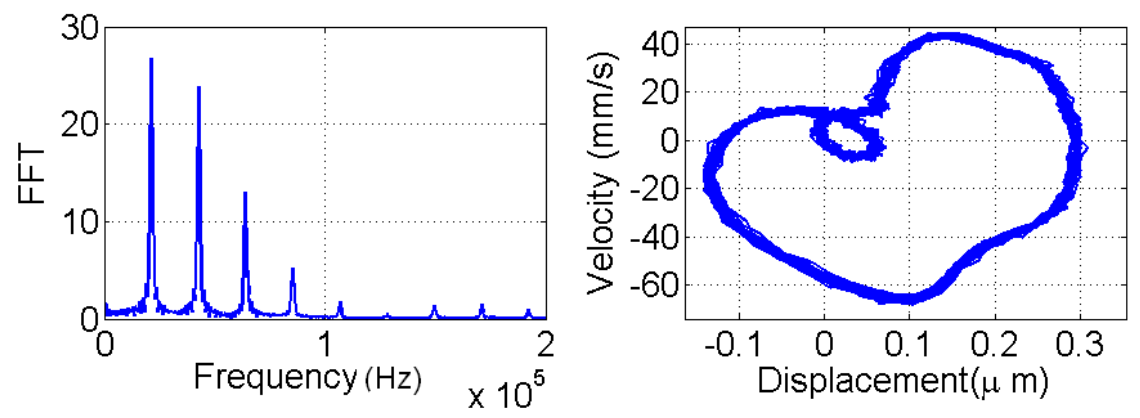

Figure 15. Fast Fourier Transform of the tip velocity and phase portrait for superharmonic resonance order 4 obtained for a beam with a natural frequency of $85 \mathrm{kHz}$ when the controller parameters are $G=2, \Psi=0.5 \mathrm{~V}$ at the excitation $v=2.78 \mathrm{~V}, \omega=21.36 \mathrm{kHz}$.

\subsection{Open Loop Superharmonic Resonances}

Superharmonic resonance was also observed in the open loop system for excitation at integer fractions of the natural frequency. The beam had the nominal dimensions of $175 \times 10 \times 2$ and a gap of $2 \mu \mathrm{m}$ with a natural frequency of $80 \mathrm{kHz}$. The DC voltage was held constant at $5 \mathrm{~V}$. Figure 16 presents the FFT of the beam tip velocity response. Superharmonic resonances of order 6 to 3 in descending order are depicted in parts (a) to (d) of this figure. The orders of the superharmonics are clear from the number of peaks in the FFT up to and including the natural frequency peak at $80 \mathrm{kHz}$.
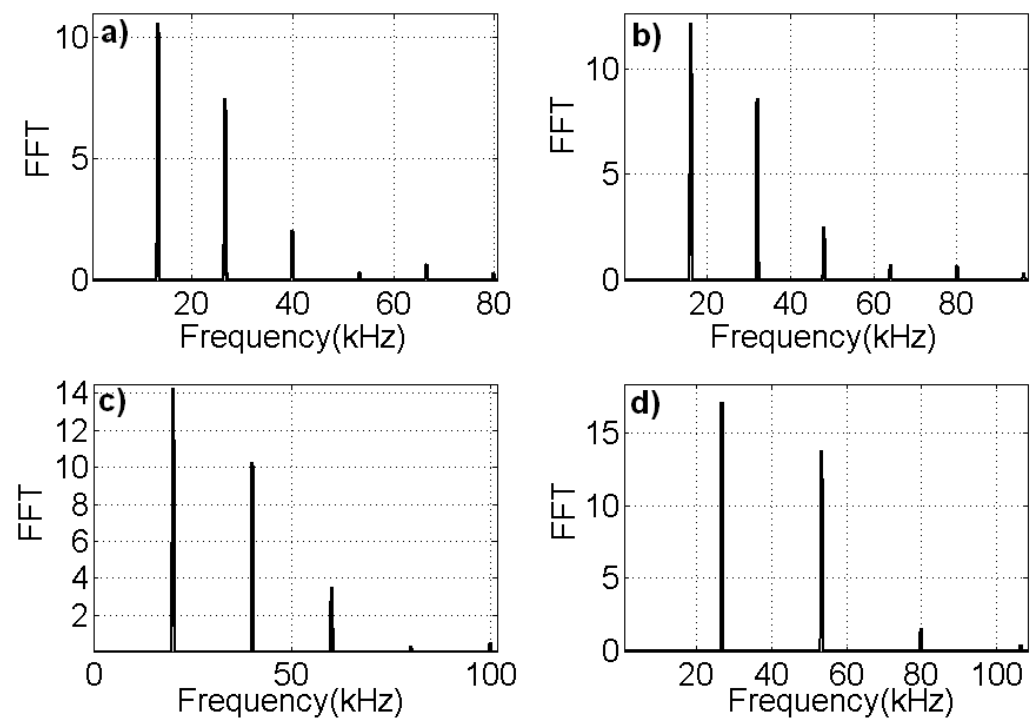

Figure 16. The superharmonic resonances of an actuator with the nominal dimensions of $175 \times 10 \times 2 \mu \mathrm{m}$ and a gap of $2 \mu \mathrm{m}$ a) order 6 obtained at $V_{D C}=5.2 \mathrm{~V}, V_{A C}=$ $4.7 \mathrm{~V}, \Omega=13.3 \mathrm{kHz}, \mathrm{b}$ ) order 5 obtained at $V_{D C}=5.2 \mathrm{~V}, V_{A C}=4.16 \mathrm{~V}, \Omega=16 \mathrm{kHz}$, c) order 4 obtained at $V_{D C}=5.2 \mathrm{~V}, V_{A C}=3.88 \mathrm{~V}, \Omega=20 \mathrm{kHz}, \mathrm{d}$ ) order 3 obtained at $V_{D C}=5.2 \mathrm{~V}, V_{A C}=3.32 \mathrm{~V}, \Omega=26.6 \mathrm{kHz}$. 
Although open loop superharmonic resonances require less equipment to produce the resonance, the closed loop superharmonics carry comparatively more power in higher harmonics as indicated by the FFT peaks for superharmonic orders of 6, 5, and 4 . The larger kinetic energy of the higher harmonics makes closed loop superharmonic resonances good candidates for applications such as secure communication devices [24], where sending and receiving a signal in a wide range of frequencies rather than a single frequency are desirable.

\section{Conclusion}

The actuation range of a closed-loop parallel-plate electrostatic actuators in attracting mode was extended in quasi-static operation mode to $90 \%$ of the capacitor gap. This is a significant improvement since the largest reported quasi-static stroke does not exceed $60 \%$ of the gap [11]. The actuator consists of a micro-cantilever beam and a voltage controller developed for low frequency applications such as trajectory-tracking and micro-positioning. The closed-loop actuator voltage requirements are moderate, in fact the total actuation voltage is less than the pull-in voltage of the open-loop actuator.

Likewise, the travel range of the open loop actuator was increased using a customized voltage waveform generated using a model of the closed loop system. Exciting the beam with the proposed waveform eliminates the need for an external feedback system, while allowing for actuation in quasi-static operation mode with a stroke as large as $75 \%$ of the gap. Such a large stroke in an open loop actuator is well beyond the present restricted quasi-static actuation range of one third of the gap. Our results show that this longstanding limitation can be overcome with careful analysis and design of open and closed loop actuators.

In the closed loop actuator, the tip velocity is measured using a laser Doppler vibrometer and the corresponding voltage signal is used to regulate the actuator voltage. For practical deployment of the actuator, the vibrometer can be replaced by a piezoresistor implanted at the root of the micro-beam to act as a displacement sensor. The actuator is then combined with the analog controller on a CMOS chip. It could be emphasized that both our open and closed loop actuators were tested successfully in an open air environment with significant external disturbances and without any requirements for a vacuum chamber.

Higher order superharmonic resonances were also observed for the first time both for open loop and closed loop electrostatic actuators. The superharmonic resonances are important observations in nonlinear MEM systems and can be used to develop sensors and communication receivers.

\section{References}

[1] B. Borovic, C. Hong, A. Q. Liu, L. Xie, and F. L. Lewis. Control of a MEMS optical switch. In 43rd IEEE Conference on Decision and Control (CDC), volume 3, pages 3039-44, Nassau, Bahamas, 2004. 
[2] F. Sugihwo, M.C. Larson, and J. S. Harris. Micromachined widely tunable vertical cavity laser diodes. Journal of Microelectromechanical Systems, 7(1):48-55, 1998.

[3] E. S. Hung. Positioning, control, and dynamics of electrostatic actuators for use in optical and RF systems. PhD thesis, Massachusetts Institute of Technology, 1998.

[4] S. Chung. Design and fabrication of $10 \times 10$ micro-spatial light modulator array for phase and amplitude modulation. Sensors and Actuators A: Physical, 78(1):63-70, 1999.

[5] P. Vettiger, G. Cross, M. Despont, U. Drechsler, U. Durig, B. Gotsmann, W. Haberle, M. A. Lantz, H.E. Rothuizen, R. Stutz, and G.K. Binnig. The "millipede" - nanotechnology entering data storage. IEEE Transactions On Nanotechnology, 1(1):39-55, 2002.

[6] J. I. Seeger and B. E. Boser. Charge control of parallel-plate, electrostatic actuators and the tip-in instability. Journal of Microelectromechanical Systems, 12(5):656-671, 102003.

[7] D. H. S. Maithripala, J. M. Berg, and W. P. Dayawansa. Control of an electrostatic microelectromechanical system using static and dynamic output feedback. Transactions of the ASME.Journal of Dynamic Systems, 127(3):443-450, 092005.

[8] R. Guardia, A. Dehe, R. Aigner, and L. M. Castaner. Current drive methods to extend the range of travel of electrostatic microactuators beyond the voltage pull-in point. Journal of Microelectromechanical Systems, 11(3):255-263, 062002.

[9] E.K. Chan and R. W. Dutton. Electrostatic micromechanical actuator with extended range of travel. Journal of Microelectromechanical Systems, 9(3):321-328, 2000.

[10] E.S. Hung and S. D. Senturia. Extending the travel range of analog-tuned electrostatic actuators. Journal of Microelectromechanical Systems, 8(4):497-505, 1999.

[11] M. S.-C. Lu and G. K. Fedder. Position control of parallel-plate microactuators for probe-based data storage. Journal of Microelectromechanical Systems, 13(5):759-769, Oct. 2004.

[12] J. Chen, W. Weingartner, A. Azarov, and R. C. Giles. Tilt-angle stabilization of electrostatically actuated micromechanical mirrors beyond the pull-in point. Journal of Microelectromechanical Systems, 13(6):988-997, 2004.

[13] C. G. Agudelo, M. Packirisamy, G. Zhu, and L. Saydy. Nonlinear control of an electrostatic micromirror beyond pull-in with experimental validation. Journal of Microelectromechanical Systems, 18(4):914-923, 2009.

[14] Y. Zhao, E H T. Francis, F. C. Siong, and G. Zhou. Stabilization of dual-axis micromirrors beyond the pull-in point by integral sliding mode control. Journal of Micromechanics and Microengineering, 16(7):1242-1250, July 2006.

[15] D. Piyabongkarn, Y. Sun, R. Rajamani, A. Sezen, and B. J. Nelson. Travel range extension of a MEMS electrostatic microactuator. IEEE Transactions on Control Systems Technology, 13(1):138-145, 012005.

[16] K. O. Owusu and F. L. Lewis. Solving the "pull-in" instability problem of electrostatic microactuators using nonlinear control techniques. In 2nd IEEE International Conference on Nano/Micro Engineered and Molecular Systems, Bangkok, Thailand, Jan. 2007.

[17] M. Nikpanah, Y. Wang, F. Lewis, and A. Liu. Real time controller design to solve the pullin instability of MEMS actuator. In 10th International Conference on Control, Automation, Robotics and Vision, Hanoi, Vietnam, Dec 2008.

[18] S. Liu, A. Davidson, and Q. Lin. Simulation studies on nonlinear dynamics and chaos in a MEMS cantilever control system. Journal of Micromechanics and Microengineering, 14(7):1064-1073, 2004.

[19] S. Towfighian, G. R. Heppler, and E. M. Abdel-Rahman. Analysis of a chaotic electrostatic microoscillator. Journal of Computational and Nonlinear Dynamics, 6(1):011001-10, Jan 2011.

[20] S. Towfighian, A. Seleim, E. M. Abdel-Rahman, and G. R. Heppler. Experimental validation for an extended stability electrostatic actuator. In Proceedings of the ASME 2010 International Design Engineering Technical Conferences, pages DETC2010-28983, Montreal, QC, Canada, 2010.

[21] A. Seleim. Design and implementation of a controller for an electrostatic MEMS actuator and 
sensor. Master's thesis, University of Waterloo, 2010.

[22] S. Towfighian. A large-stroke electrostatic micro-actuator. PhD thesis, University of Waterloo, 2010.

[23] M. I. Younis and F. Alsaleem. Exploration of new concepts for mass detection in electrostaticallyactuated structures based on nonlinear phenomena. Journal of Computational and Nonlinear Dynamics, 4(2):021010-1-15, 2009.

[24] Y. C. Wang, S. G. Adams, J. S. Thorp, N. C. MacDonald, P. Hartwell, and F. Bertsch. Chaos in MEMS, parameter estimation and its potential application. IEEE Transactions on Circuits and Systems I: Fundamental Theory and Applications, 45(10):1013-1020, 1998. 